\title{
Measurement of the dynamo effect in a plasma*
}

\author{
H. $\mathrm{Ji}^{\dagger, \mathrm{a})}$ \\ Plasma Physics Laboratory, Princeton University, P.O. Box 451, Princeton, New Jersey 08543 \\ S. C. Prager, A. F. Almagri, and J. S. Sarff \\ Department of Physics, University of Wisconsin, Madison, Wisconsin 53706 \\ Y. Yagi, Y. Hirano, and K. Hattori \\ Plasma Section, Electrotechnical Laboratory, Tsukuba, Ibaraki 305, Japan \\ H. Toyama \\ Department of Physics, Faculty of Science, University of Tokyo, Bunkyo-ku, Tokyo 113, Japan
}

(Received 6 November 1995; accepted 21 November 1995)

\begin{abstract}
A series of detailed experiments has been conducted in three laboratory plasma devices to measure the dynamo electric field along the equilibrium field line (the $\alpha$ effect) arising from the correlation between the fluctuating flow velocity and magnetic field. The fluctuating flow velocity is obtained from probe measurement of the fluctuating $\mathbf{E} \times \mathbf{B}$ drift and electron diamagnetic drift. The three major findings are the following: (1) The $\alpha$ effect accounts for the dynamo current generation, even in the time dependence through a "sawtooth" cycle; (2) at low collisionality the dynamo is explained primarily by the widely studied pressureless magnetohydrodynamic (MHD) model, i.e., the fluctuating velocity is dominated by the $\mathbf{E} \times \mathbf{B}$ drift; (3) at high collisionality, a new "diamagnetic dynamo" is observed, in which the fluctuating velocity is dominated by the electron diamagnetic drift. In addition, direct measurements of the helicity flux indicate that the dynamo activity transports magnetic helicity from one part of the plasma to another, but the total helicity is roughly conserved, verifying Taylor's [Phys. Rev. Lett. 33, 1139 (1974); Rev. Mod. Phys. 58, 741 (1986)] conjecture. C 1996 American Institute of Physics. [S1070-664X(96)90105-9]
\end{abstract}

\section{INTRODUCTION}

Creation or amplification of the magnetic flux by plasma dynamics, often called a dynamo effect, is considered as one of the most important physical phenomena in astrophysical and laboratory plasmas. The latter are only examples in which the dynamo effect can be actively controlled and directly measured experimentally. The reversed-field-pinch (RFP) toroidal plasma, in which the toroidal field reverses its direction at the edge, is a particularly vivid example of the dynamo effect. In the RFP, the externally applied electric field is in the toroidal direction. Thus, the poloidal current near the edge, essentially parallel to the magnetic field, is generated and maintained by the dynamo electric field against resistive diffusion.

In the most widely studied magnetohydrodynamic (MHD) dynamo model, a fluctuation-induced electromotive electric field $\langle\tilde{\mathbf{v}} \times \tilde{\mathbf{B}}\rangle_{\|}$sustains the field-aligned current against resistive decay in the parallel Ohm's law ${ }^{1}$

$$
E_{\|}+\langle\tilde{\mathbf{v}} \times \tilde{\mathbf{B}}\rangle_{\|}=\eta j_{\|},
$$

where $E_{\|}$is the equilibrium electric field parallel to the magnetic field, $\eta$ the electric resistivity, $j_{\|}$the parallel equilibrium current, $\tilde{\mathbf{v}}$ and $\tilde{\mathbf{B}}$ are the fluctuating fluid velocity and magnetic field respectively, and $\langle\ldots\rangle$ denotes an average over an equilibrium flux surface. This model has been intensively employed in analytical theories ${ }^{1}$ as well as in numerical

\footnotetext{
*Paper 71A, Bull. Am. Phys. Soc. 40, 1803 (1995).

${ }^{\dagger}$ Invited speaker.

${ }^{a)}$ Electronic mail: hji@pppl.gov
}

simulations both for the general astrophysical dynamo problems $^{2}$ and for the specific RFP configuration. ${ }^{3}$

As a phenomenon, the dynamo effect has been identified in the RFP plasmas, ${ }^{4}$ spheromak plasmas ${ }^{5}$ as well as in liquid metals. ${ }^{6}$ However, it was not until recently ${ }^{7}$ that the direct observation of the dynamo electric field has been attempted by measuring $\tilde{\mathbf{v}}$ and $\tilde{\mathbf{B}}$ simultaneously, followed by other experiments. ${ }^{8-10}$ In this paper, we report the results obtained by a series of the detailed experiments, ${ }^{7,11,12}$ conducted in three RFP devices. MHD dynamo has been observed in low collisionality region while a new "diamagnetic dynamo" has been observed in the high collisionality region. In addition to the continuous dynamo effect, a discrete dynamo electric field has been detected during "sawtooth crash" phase of plasma evolution. These results will be described in Sec. IV and Sec. V.

Arrangement for other sections is the following. In Sec. II, a brief derivation of the parallel Ohm's law in a turbulent plasma is given in order to identify possible dynamo terms. In Sec. III, experimental apparatus including three RFP devices and measuring probe schemes are described. After the presentation of the main results in Secs. IV and V, interpretation of the results and discussions will be given in Sec. VI, followed by conclusions in Sec. VII. Description of the data analysis methods are attached as an appendix.

\section{PARALLEL OHM'S LAW IN A TURBULENT PLASMA}

We start from the generalized Ohm's law, ${ }^{13}$

$$
-\frac{m_{e}}{e^{2} n} \frac{\partial \mathbf{j}}{\partial t}+\mathbf{E}+\mathbf{v} \times \mathbf{B}-\frac{1}{e n} \mathbf{j} \times \mathbf{B}+\frac{\boldsymbol{\nabla} P_{e}}{e n}=\eta \mathbf{j},
$$


where $n$ is the electron density and $P_{e}$ the electron pressure. By splitting every quantity into mean (denoted by subscript 0 ) and fluctuating (denoted by tildes) parts, averaging over a flux surface, and taking the parallel component, the parallel Ohm's law in a turbulent plasma becomes

$$
\eta_{\|} j_{\| 0}-E_{\| 0}=\langle\tilde{\mathbf{v}} \times \tilde{\mathbf{B}}\rangle_{\|}-\langle\tilde{\mathbf{j}} \times \tilde{\mathbf{B}}\rangle_{\|} / e n,
$$

where we have neglected three small terms $\partial j \| / \partial t,\left\langle\tilde{\eta} \tilde{j}_{\|}\right\rangle$ and $\left\langle\tilde{n} \nabla_{\|} \tilde{P}_{e}\right\rangle / e n^{2}$, as appropriate for the experimental condition. The right-hand side (RHS) includes the usual $\langle\tilde{\mathbf{v}} \times \tilde{\mathbf{B}}\rangle$ term and the Hall term. Since $\mathbf{v}=\left(m_{i} \mathbf{v}_{i}+m_{e} \mathbf{v}_{e}\right) /$ $\left(m_{i}+m_{e}\right) \approx \mathbf{v}_{i}$ and $\mathbf{j}=e n\left(\mathbf{v}_{i}-\mathbf{v}_{e}\right)$, Eq. (3) can be rewritten as

$$
\eta_{\|\|_{\| 0}}-E_{\| 0}=\langle(\tilde{\mathbf{v}}-\tilde{\mathbf{j}} / e n) \times \tilde{\mathbf{B}}\rangle_{\|} \approx\left\langle\tilde{\mathbf{v}}_{e} \times \tilde{\mathbf{B}}\right\rangle_{\|},
$$

where $\mathbf{v}_{i}\left(\mathbf{v}_{e}\right)$ is the ion (electron) flow velocity. We note that the appearance of $\mathbf{v}_{e}$ only in the RHS is consistent with the parallel Ohm's law being a force balance of electrons.

An alternative form of the parallel Ohm's law can be derived by substituting the perpendicular component of Eq. (2),

$$
\tilde{\mathbf{v}}_{e \perp} \approx \tilde{\mathbf{v}}_{\perp}-\tilde{\mathbf{j}}_{\perp} / e n \approx\left(\tilde{\mathbf{E}}_{\perp} \times \mathbf{B}_{0}+\nabla_{\perp} \tilde{P}_{e} \times \mathbf{B}_{0} / e n\right) / B^{2},
$$

into Eq. (4) to yield

$$
\eta_{\|\|_{\| 0}}-E_{\| 0}=\left\langle\tilde{\mathbf{E}}_{\perp} \cdot \tilde{\mathbf{b}}_{\perp}\right\rangle+\left\langle\nabla_{\perp} \tilde{P}_{e} \cdot \tilde{\mathbf{b}}_{\perp}\right\rangle / e n,
$$

where $\mathbf{b} \equiv \mathbf{B} / B$.

We identify two possible dynamo terms in the RHS of Eq. (6). The first term $\left\langle\tilde{\mathbf{E}}_{\perp} \cdot \tilde{\mathbf{b}}_{\perp}\right\rangle$, represents the contribution to $\tilde{\mathbf{v}}_{e \perp}$ from the fluctuating $\tilde{\mathbf{E}}_{\perp} \times \mathbf{B}_{0}$ drift which is a MHD (single fluid) effect, while the second term, $\left\langle\nabla_{\perp} \tilde{P}_{e} \cdot \tilde{\mathbf{b}}_{\perp}\right\rangle / e n$, is the contribution from the fluctuating electron diamagnetic drift $\nabla_{\perp} \tilde{P}_{e} \times \mathbf{B}_{0}$ which is an electron fluid effect (in the two-fluid framework). (It should be clarified here that the latter is different from the so-called "battery effect" 14 in Faraday's induction law, which involves no magnetic fluctuations in the early growing phase of the dynamo field.) We emphasize here that only the $\mathbf{E} \times \mathbf{B}$ effect has been incorporated in most MHD computations ${ }^{2,3}$ where the total plasma pressure has usually been set to zero.

The aim of the present experiments is to identify the dynamo mechanism by measuring both the MHD dynamo term, $\left\langle\tilde{\mathbf{E}}_{\perp} \cdot \tilde{\mathbf{b}}_{\perp}\right\rangle \approx\left\langle\tilde{E}_{t} \tilde{b}_{t}\right\rangle+\left\langle\tilde{E}_{r} \tilde{b}_{r}\right\rangle$, and the diamagnetic dynamo term, $\left\langle\boldsymbol{\nabla}_{\perp} \tilde{P}_{e} \cdot \tilde{\mathbf{b}}_{\perp}\right\rangle \approx\left\langle\left(\boldsymbol{\nabla}_{t} \tilde{P}_{e}\right) \tilde{b}_{t}\right\rangle+\left\langle\left(\boldsymbol{\nabla}_{r} \tilde{P}_{e}\right) \tilde{b}_{r}\right\rangle$ in the RFP edge, where the poloidal field $B_{p}$ dominates the toroidal field $B_{t}$. Here the subscripts $t$ and $r$ denote the toroidal and radial components, respectively.

\section{EXPERIMENTAL APPARATUS}

The experiments described here were carried out in three RFP devices: Madison Symmetric Torus (MST) ${ }^{15}$ Reversed Field Pinch University of Tokyo Experiments (REPUTE) ${ }^{16}$ and Toroidal Pinch Experiment (TPE-1RM20). ${ }^{17}$ MST is a large sized RFP while REPUTE and TPE-1RM20 are medium sized RFP devices. Table I lists important parameters of these three devices. Measurements were performed in the relatively low plasma current discharges to avoid heat damage to the inserted probes. All measurements were taken around the current flattop period, in which MST discharges
TABLE I. Major parameters of three RFP plasmas: MST, REPUTE and TPE-1RM20. Also listed is local plasma parameters where the dynamo measurements took place.

\begin{tabular}{lccc}
\hline \hline Device & MST & REPUTE & TPE \\
\hline$R(\mathrm{~m})$ & 1.50 & 0.82 & 0.75 \\
$a(\mathrm{~m})$ & 0.51 & 0.22 & 0.192 \\
$I_{p}(\mathrm{kA})$ & $210 / 130$ & 110 & 50 \\
$V_{\text {loop }}(\mathrm{V})$ & $20 / 20$ & 220 & $20-45$ \\
$\bar{n}_{e}\left(10^{19} / \mathrm{m}^{3}\right)$ & $1.1 / 0.6$ & 4.4 & $0.4-1.9$ \\
$T_{e}(0)(\mathrm{eV})$ & $120 / 100$ & $\sim 50$ & $\sim 100$ \\
\hline$r / a$ & $0.90 / 0.92$ & 0.85 & 0.92 \\
$T_{e}(\mathrm{eV})$ & $30 / 15$ & $\sim 8$ & $10-20$ \\
$n_{e}\left(10^{18} / \mathrm{m}^{3}\right)$ & $2 / 1.6$ & 10 & $2-10$ \\
$\lambda_{e}(\mathrm{~m})$ & $2.8 / 1.0$ & $\sim 0.04$ & $0.08-0.8$ \\
$\lambda_{e} / a$ & $5.5 / 1.9$ & $\sim 0.2$ & $0.4-4$ \\
\hline \hline
\end{tabular}

typically show the discrete sawtooth oscillations with several millisecond repetition period ${ }^{18}$ while REPUTE and TPE discharges contain rather irregular oscillations.

The major diagnostics used here include two versions of a "complex Langmuir probe"19 (Fig. 1) and an insertable Rogowskii coil probe ${ }^{20}$ (with the outer diameter of $3 \mathrm{~cm}$ ) which measures the local poloidal (parallel) current. Each version of the complex probe consists of two triple probes to measure electron temperature $T_{e}$, density $n$, and floating potential $V_{f}$ at two locations separated by $1.27 \mathrm{~cm}$ toroidally (in the toroidal version) or $0.25 \mathrm{~cm}$ radially (in the radial version.) The toroidal version of the complex probe has been modified to block the fast electrons ${ }^{21-23}$ from the tungsten or molybdenum tips with a small boron nitride obstacle while the radial version has been aligned so that the tips face away
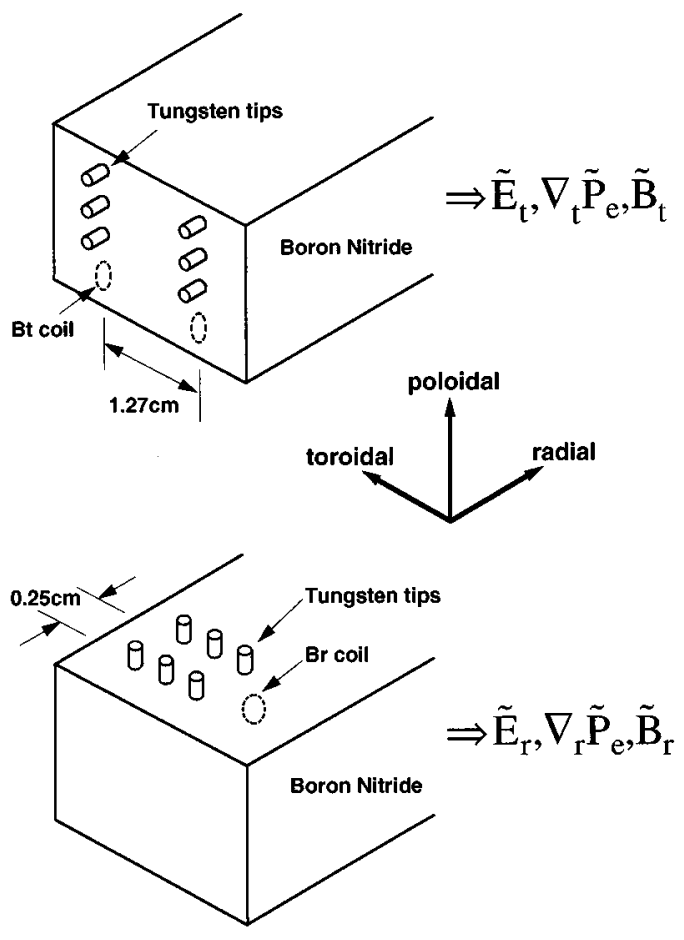

FIG. 1. Schematic view of two versions of the complex probe. 


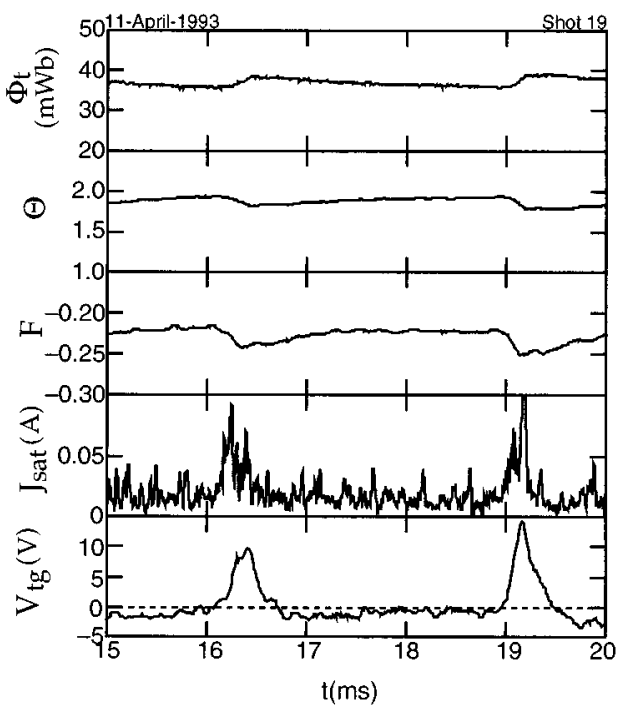

FIG. 2. Waveforms of toroidal flux $\Phi_{t}$, pinch parameter $\Theta$, reversal parameter $F$, ion saturation current $J_{\text {sat }}$ measured by the triple probe at $r / a=0.98$, and voltage across toroidal gap in the shell $V_{\text {tg }}$ during two sawtooth oscillations.

from fast electrons. Thus the fast electron effects on probe measurements are eliminated for the entire range of density.

The electrostatic components of electric fields $E_{t}$ and $E_{r}$ are obtained from the difference in plasma potential $V_{p}=V_{f}+c T_{e}$, where $c \simeq 2.5(0.8)$ for $E_{t}\left(E_{r}\right)$ calculated from the electron-ion collection area ratio at the different orientation of the probe tips with respect to the magnetic field. ${ }^{19}$ The inductive components of the perpendicular electric field fluctuations are negligible. Similarly, the fluctuations in gradient of the electron pressure are obtained from spatial differences; $B_{t}$ and $B_{r}$ and their fluctuations are measured by the magnetic pick-up coils installed in the complex probes.

\section{OBSERVATION OF DISCRETE AND CONTINUOUS MHD DYNAMO}

Sawtooth oscillations in MST represent discrete dynamo events. Figure 2 illustrates field generation and relaxation over two sawtooth oscillations spanning $5 \mathrm{~ms}$. Strong spontaneous field generation is evident in the sudden increase of the toroidal flux $\Phi_{t}$ during a sawtooth crash (in $\sim 0.1 \mathrm{~ms}$ ). Between crashes, flux generation (opposing resistive decay) is present but mild. The decreases in the pinch parameter $\Theta \equiv B_{p}(a) /\left(\Phi_{t} / \pi a^{2}\right)$ and the reversal parameter $F \equiv B_{t}(a) /\left(\Phi_{t} / \pi a^{2}\right)$ show that the plasma relaxes toward the minimum energy state with a flatter current profile, i.e., current decreases at the core and increases at the edge. The edge density and electron temperature also increase during a crash, as represented in Fig. 2 by the ion saturation current $J_{\text {sat }}$ measured by the triple probe at $r / a=0.98$. The time derivative of the toroidal flux, measurable as the voltage across the toroidal gap in the shell, $V_{\text {tg }}$, is employed as a time reference for the sawtooth crash.

A time-domain method has been developed to calculate correlation, coherence and phase difference between two
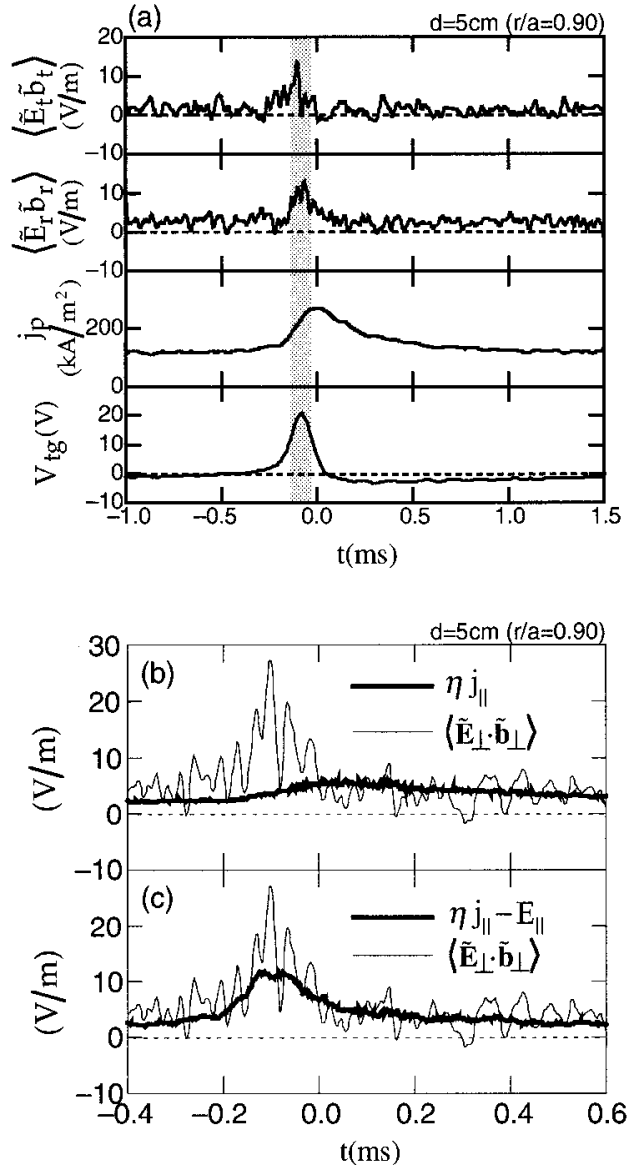

FIG. 3. (a) Ensemble-averaged MHD dynamo electric fields and local parallel current density during one sawtooth crash, measured at $r / a=0.90$. The toroidal gap voltage, $V_{\text {tg }}$, marks the timing of the sawtooth crash. MHD dynamo electric field $\left\langle\tilde{\mathbf{E}}_{\perp} \cdot \tilde{\mathbf{b}}_{\perp}\right\rangle$ is compared to $\eta j_{\|}$(b) and $\eta j_{\|}-E_{\|}$(c).

fluctuations for the time-dependent phenomena, instead of the conventional spectral analysis. Details of the method are described in the Appendix. The samples are taken from 30 identical discharges and 150 sawtooth crashes with the plasma current $I_{p} \simeq 210 \mathrm{kA}$ and the line-averaged density $\bar{n}_{e} \simeq 1.1 \times 10^{19} / \mathrm{m}^{3}$. Fluctuation amplitudes peak at the sawtooth crash while the coherence $(\simeq 0.1)$ and the phase difference $(\simeq 0$, in phase $)$ between $\tilde{\mathbf{E}}_{\perp}$ and $\tilde{\mathbf{B}}_{\perp}$ remain unchanged. ${ }^{11}$ The two components of $\left\langle\tilde{\mathbf{E}}_{\perp} \cdot \tilde{\mathbf{b}}_{\perp}\right\rangle$ measured at $r / a=0.90$ are shown in Fig. 3(a). Both $\left\langle\tilde{E}_{t} \tilde{b}_{t}\right\rangle$ and $\left\langle\tilde{E}_{r} \tilde{b}_{r}\right\rangle$ peak during the crash. The local poloidal current density $j_{p}$ keeps rising during the crash and peaks at the end of the crash, consistent with current profile flattening.

To establish the strength of the MHD dynamo term we compare it to other measured terms in Ohm's law [Eq. (6)]. In Fig. 3(b), we compare the measured MHD dynamo electric field to the resistive term $\eta j_{\|}$, where $\eta$ is Spitzer's resistivity calculated from the measured local $T_{e}$ but estimated $Z_{\text {eff }}=2$. In spite of large experimental error bars, fairly good agreement can be seen between $\left\langle\tilde{\mathbf{E}}_{\perp} \cdot \tilde{\mathbf{b}}_{\perp}\right\rangle$ and $\eta j_{\|}$except for the burst of dynamo electric field during the crash. The electric field term $E_{\|}$is small for the steady state case but can be large during the sawtooth crash because of its transient nature. The parallel electric field at the edge is given by 


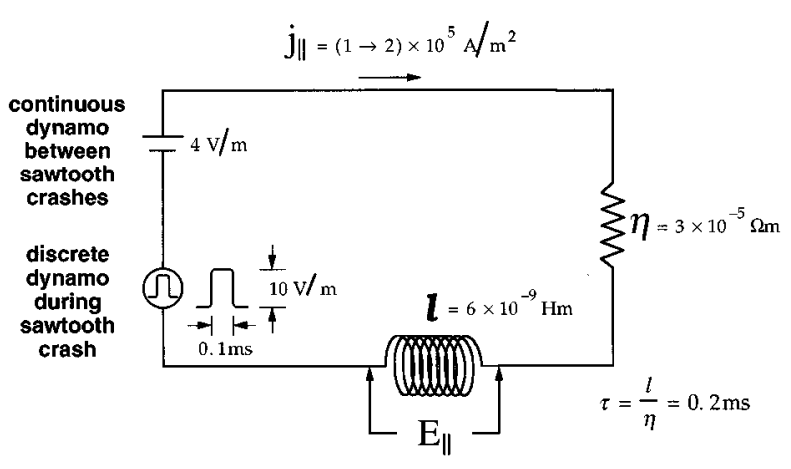

FIG. 4. A simple electric circuit as an analogy of the observed continuous and discrete MHD dynamo electric field during the sawtooth cycle in MST. The effective $\mathrm{L} / \mathrm{R}$ time constant $\sim 0.2 \mathrm{~ms}$, consistent with the decay time of $j_{\|}$after the sawtooth crash.

$$
E_{\|}(r) \approx\left(V_{\mathrm{tg}}-2 \pi \int_{r}^{a} \dot{B}_{t} r \mathrm{~d} r\right) / 2 \pi r,
$$

where the first term dominates. By including the electric field, Fig. 3(c) shows good agreement between $\left\langle\tilde{\mathbf{E}}_{\perp} \cdot \tilde{\mathbf{b}}_{\perp}\right\rangle$ and $\eta j_{\|}-E_{\|}$at all times within experimental uncertainty. Contribution from the fluctuating electron diamagnetic drift [i.e., the second term in the RHS of Eq. (6)] has been measured to be small [see Fig. 6(b) below].

The observed MHD dynamo can be summarized as in Fig. 4: (a) a continuous dynamo electric field drives equilibrium poloidal current between the sawtooth crashes and (b) the crash generates a burst of (discrete) dynamo electric field which is largely balanced by an inductive $E_{\|}$during the $j_{\|}$ rising phase. The effective inductance can be estimated as $l=E_{\|} /(\mathrm{d} j \| / \mathrm{d} t) \sim 6 \times 10^{-9} \mathrm{Hm}$. The resulting $L / R$ time constant is $\tau=l / \eta \sim 0.2 \mathrm{~ms}$, consistent with the decay time of $j_{\|}$after the sawtooth crash.

\section{OBSERVATION OF DIAMAGNETIC DYNAMO}

In an earlier experiment ${ }^{7}$ in REPUTE RFP, the measured MHD dynamo electric field was far below that required to balance resistive dissipation, as shown in Fig. 5, where the data were taken in $I_{p} \simeq 110 \mathrm{kA}, \bar{n}_{e} \simeq 4.4 \times 10^{19} / \mathrm{m}^{3}$ plasmas. One of the most distinct differences between MST and REPUTE RFPs is that the MST edge is much more collisionless than REPUTE. One way to measure the collisionality is to calculate the ratio of electron mean free path $\lambda_{e}$ to the plasma size, say, the minor radius $a$. This ratio in MST edge is 2-7 compared 0.08-0.4 in the REPUTE edge. The current MST operation ${ }^{15}$ is limited to the relatively low density region presumably due to its large size, $R / a=1.50 \mathrm{~m} / 0.52 \mathrm{~m}$, while the low current discharges in REPUTE was limited to the high density (see Table I). Thus an important question still remains whether the MHD dynamo model is valid in general or limited to only certain conditions.

In order to answer this question, the third RFP device, TPE-1RM20, has been employed to perform the collisionality scan. In the normal TPE operation for a fixed $I_{p}$, the upper limit of the line-averaged density $\bar{n}_{e}$ is primarily determined ${ }^{24}$ by the pinch parameter $\Theta$. Typically, $\bar{n}_{e}$ ranges from $\simeq 0.44 \times 10^{19} / \mathrm{m}^{3}$ at $\Theta \simeq 1.5$ to $\simeq 1.01 \times 10^{19} / \mathrm{m}^{3}$ at

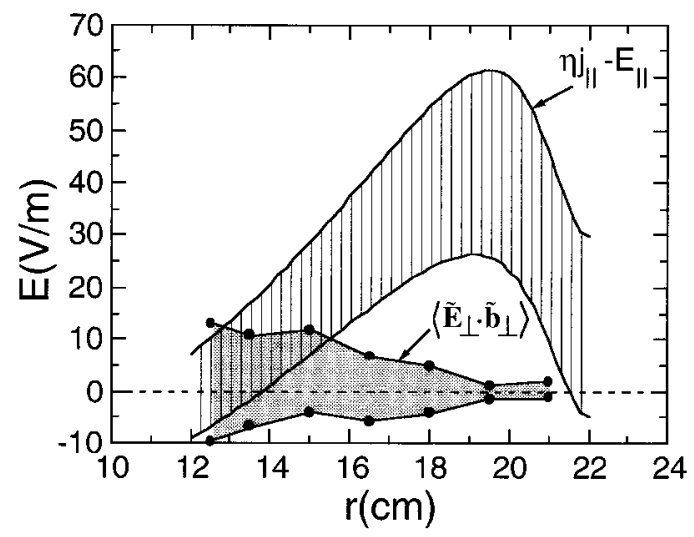

FIG. 5. Comparison between radial profile of $\eta j_{\|}-E_{\|}$and MHD dynamo electric field measured in REPUTE RFP.

$\Theta \simeq 2.0$, in $I_{p} \simeq 50 \mathrm{kA}$ plasmas. A higher density of $\bar{n}_{e} \simeq 1.86 \times 10^{19} / \mathrm{m}^{3}$ was achieved at the relatively high $\Theta \simeq 1.9$ by adding 15 wall loading discharges with the same working gas $\left(\mathrm{D}_{2}\right)$ before each main RFP discharge. By varying $\bar{n}_{e}$, the edge density at $r / a=0.92$ increases by a factor of $\simeq 4$ while the electron temperature decreases by $\simeq 35 \%$, yielding a factor of 10 change from $\sim 0.4$ to $\sim 4$ in the collisionality $\lambda_{e} / a$.

The coherences of $\left\langle\tilde{\mathbf{E}}_{\perp} \cdot \tilde{\mathbf{b}}_{\perp}\right\rangle$ and $\left\langle\boldsymbol{\nabla}_{\perp} \tilde{P}_{e} \cdot \tilde{\mathbf{b}}_{\perp}\right\rangle /$ en are shown in Fig. 6(a) for four different densities. The coherence for both terms is comparable at the low density cases. When the density increases, however, coherence in the MHD dynamo term (the solid curves) decreases nearly to the statistical confidence level determined by the number of samples in the ensemble $(1 / \sqrt{N})$. On the other hand, coherence in the diamagnetic dynamo term (the dotted curves) remains roughly constant. The relative phase angle is $\sim 0$ (in phase) for all cases and changes little with density. As a result, the MHD dynamo term dominates over the diamagnetic dynamo term for the three relatively low density cases while the latter becomes larger for the highest density case. ${ }^{12}$ This relative variation arises mainly from changes in the coherence as well as in the fluctuation levels.

Figure 7(a) compares the dynamo electric fields with the resistive term $\eta j$ for four different density discharges. Note $E_{\|} \approx E_{p}=0$ in the steady state. For the three relatively low density cases, the MHD dynamo alone is sufficient to account for the resistive term, confirming the MHD dynamo hypothesis. However, in the highest density case the MHD dynamo diminishes while the diamagnetic dynamo becomes dominant. The sum of the two terms is large enough to account for the $\eta j$ term within error bars. Contribution of the fast electrons to the electron diamagnetic term, i.e., $\left\langle\nabla_{\perp} \tilde{P}_{e}^{\text {fast }} \cdot \tilde{\mathbf{b}}_{\perp}\right\rangle / e n$, is expected to be insignificant since the fast electron density is only a few percent of the bulk density. ${ }^{23}$

The observation in TPE unites the apparently contradictory measurements in $\operatorname{REPUTE}^{7}$ and MST. ${ }^{11}$ Figure 6(b) displays the coherences of the dynamo fields measured in the MST edge. The samples are taken from 36 identical discharges with $I_{p} \simeq 130 \mathrm{kA}$ and $\bar{n}_{e} \simeq 6.2 \times 10^{18} / \mathrm{m}^{3}$. As in the low density case of TPE, the MHD dynamo term dominates 


$$
-\left\langle\tilde{\mathbf{E}}_{\perp} \cdot \tilde{\mathbf{b}}_{\perp}\right\rangle \cdots\left(\nabla_{\perp} \tilde{\mathrm{P}}_{\mathrm{e}} \cdot \tilde{\mathbf{b}}_{\perp}\right\rangle / \mathrm{en}
$$
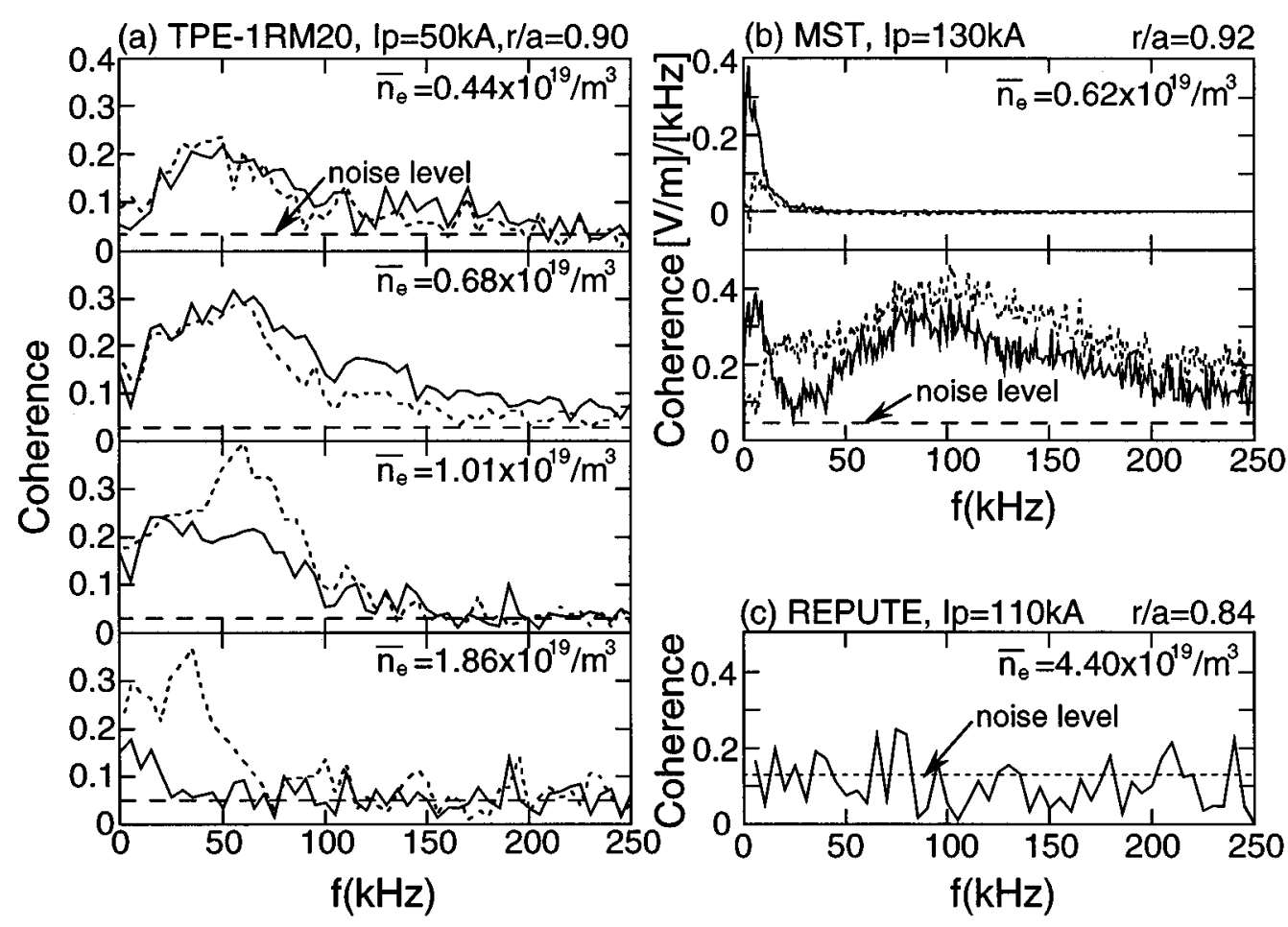

(c) REPUTE, Ip=110kA $\mathrm{r} / \mathrm{a}=0.84$

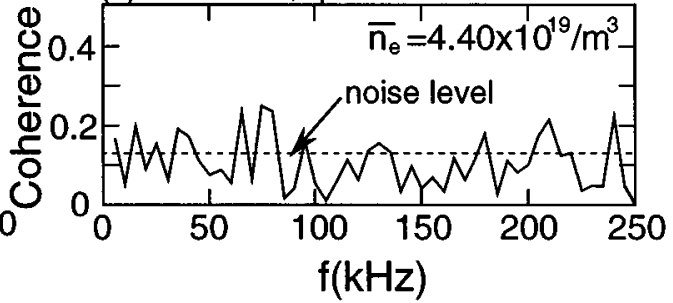

FIG. 6. (a) Coherences for the MHD dynamo term and the electron diamagnetic dynamo term in TPE-1RM20 for four different densities, (b) cross-spectra and coherences in MST, (c) coherence for the MHD dynamo term in REPUTE.

over the diamagnetic dynamo term. On the other hand, no coherent MHD dynamo is detected ${ }^{7}$ in the high density REPUTE plasmas $\left(I_{p} \simeq 110 \mathrm{kA}\right.$ and $\left.\bar{n}_{e} \simeq 4.4 \times 10^{19} / \mathrm{m}^{3}\right)$, as shown in Fig. 6(c), consistent with the TPE observations.

Thus a systematic dependence of the dynamo electric fields on the collisionality emerges from all three RFPs. A summary is given in Fig. 7(b) where the dynamo fields and their resistive terms (normalized by $E_{0}=V_{\text {loop }} / 2 \pi R$ ) are plotted against the collisionality which is varied by more than a factor of 30 . Clearly, in the collisionless region $\left(\lambda_{e} / a \gtrsim 1\right)$, the MHD dynamo is the main driver of the parallel current, while in the collisional region $\left(\lambda_{e} / a \leqslant 1\right)$, the electron diamagnetic dynamo term becomes dominant. Following this categorization, the ZETA plasma ${ }^{25}$ falls into the collisional region while other RFP plasmas, such as ZT-40M, ${ }^{26}$ fall into the collisionless region where the MHD dynamo should dominate, as marked in Fig. 7(b).

\section{DISCUSSION}

\section{A. Interpretation of the observations}

We can interpret the results via either Eq. (3) or Eq. (6). At low collisionality, the MHD dynamo dominates. Hence the $\langle\tilde{\mathbf{v}} \times \tilde{\mathbf{B}}\rangle$ term is large in Eq. (3). The cross-field flow $\tilde{\mathbf{v}}_{\perp}$ establishes an electric field $\tilde{\mathbf{E}}_{\perp}$ self-consistently through charge separation. As a result, the dynamo field $\tilde{\mathbf{v}}_{\perp} \times \tilde{\mathbf{B}}_{\perp}=\tilde{\mathbf{E}}_{\perp} \cdot \tilde{\mathbf{B}}_{\perp} / B_{0}$ is large in Eq. (6). Both electrons and ions move together and the Hall term $(\mathbf{j} \times \mathbf{B}$ term) in Eq. (3) is small, consistent with MST measurements. ${ }^{27}$
At high collisionality, the electron pressure term in Eq. (6) is large. Fluctuations in the electron pressure gradient (instead of the electric field) sustain the fluctuating electron flow velocity self-consistently. This effect would be manifest in Eq. (3) as a Hall dynamo arising from the fluctuating electron diamagnetic current $\tilde{\mathbf{j}}_{e \perp}=\mathbf{B}_{0} \times \nabla \tilde{P}_{e} / B_{0}^{2}$. The ion flow is unspecified. If one assumes strong coupling between electrons and ions, i.e., $\tilde{P}_{e} \approx \tilde{P}_{i}$, as likely in the collisional limit, then the ion diamagnetic drift $\tilde{\mathbf{v}}_{i \perp}\left(=-\nabla_{\perp} \tilde{P}_{i}\right.$ $\times \mathbf{B}_{0} /$ en $B_{0}^{2}$ ) is opposite to the electron diamagnetic drift, resulting in an anti-dynamo effect in the $\tilde{\mathbf{v}} \times \tilde{\mathbf{B}} \approx \tilde{\mathbf{v}}_{i} \times \tilde{\mathbf{B}}$ term in Eq. (3). However, this is offset by an additional dynamo effect in the Hall term from the associated ion diamagnetic current $\tilde{\mathbf{j}}_{i \perp}=\mathbf{B}_{0} \times \nabla \tilde{P}_{i} / B_{0}^{2}$.

We suggest two possible physical reasons for the transition by collisions. First, an increase in the perpendicular conductivity with collisions can suppress the electric field. Second, the collisions could reduce $\tilde{\mathbf{v}}_{i \perp}$ through the ion perpendicular viscosity $\nu_{i \perp} \propto n^{2} /{\sqrt{T_{i}}}^{28}$ The differential perpendicular electron and ion flows result in a perpendicular current $\tilde{\mathbf{j}}_{\perp}$ which establishes the pressure gradient by $\tilde{\mathbf{j}}_{\perp} \times \mathbf{B}_{0}$ force in a self-consistent way. In any case, as implied by Eq. (4), the dynamo is carried out by electron dynamics only.

\section{B. Implication for the dynamo theories}

Our results clearly support validity of the MHD dynamo model in the collisionless region. Alternatively, the kinetic 


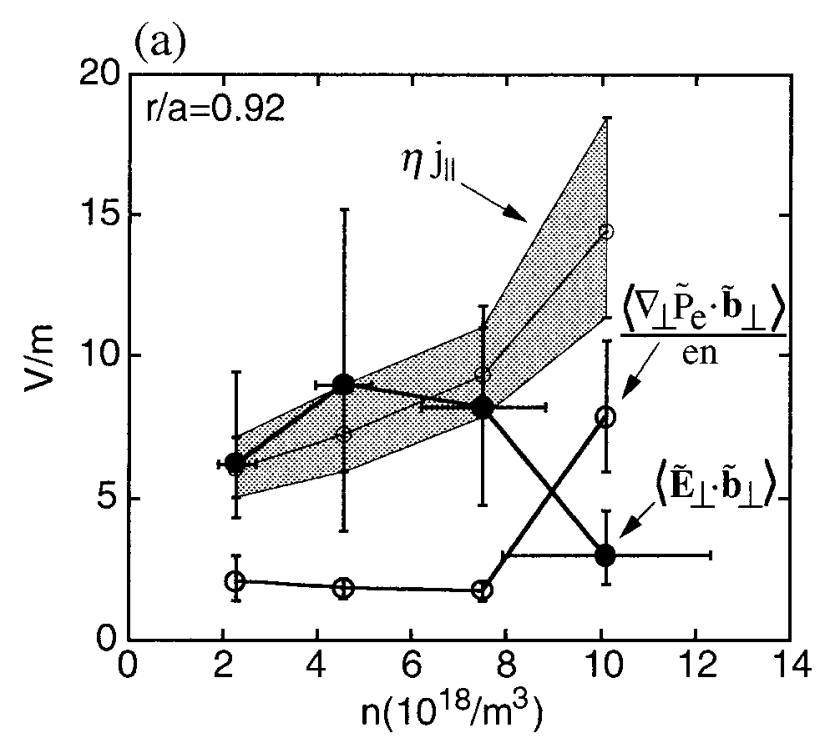

(b)

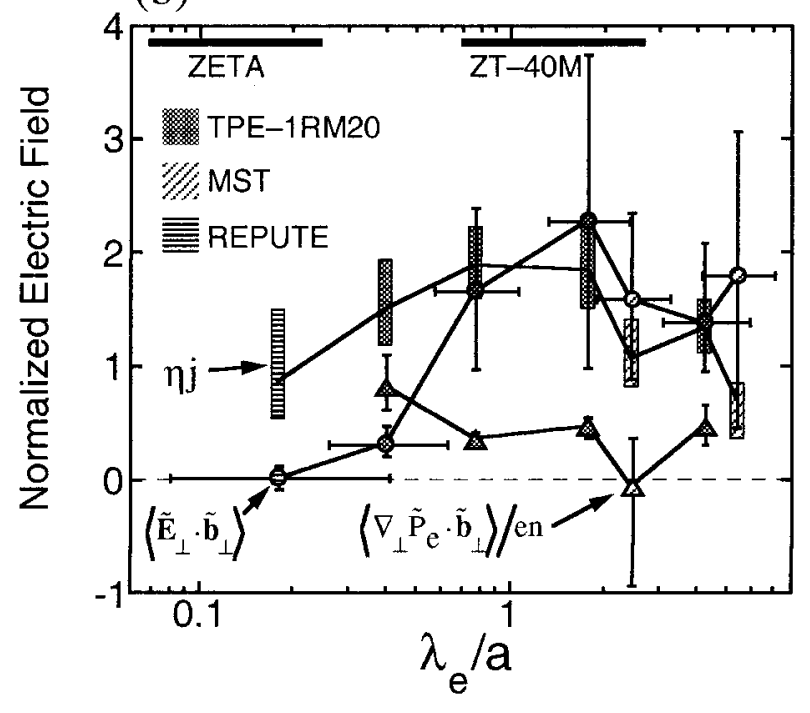

FIG. 7. (a) Comparison of the dynamo terms to the resistive term $\eta j$ as a function of the local density in TPE-1RM20. (b) Normalized dynamo terms and resistive term $\eta j$ versus normalized electron mean free path in the edge of TPE, MST and REPUTE plasmas. Also shown is the collisionality ranges for the ZETA and ZT-40M edge.

dynamo theory $(\mathrm{KDT})^{29}$ has been proposed to explain the RFP dynamo effect. The KDT is based on radial diffusion of the parallel current due to a prescribed stochastic magnetic field and it is expected to be activated in the collisionless region. However, the observation of collisionless MHD dynamo implies the ineffectiveness of the KDT mechanism. On the other hand, the observation is consistent with the TerryDiamond theory ${ }^{30}$ which incorporates self-consistent constraints and predicts negligible kinetic dynamo effect in the collisionless limit.

By rewriting the diamagnetic dynamo term as

$$
\left\langle\nabla_{\perp} \tilde{P}_{e} \cdot \tilde{\mathbf{b}}_{\perp}\right\rangle \approx \nabla \cdot\left\langle\tilde{P}_{e} \tilde{\mathbf{b}}\right\rangle,
$$

the quantity $\left\langle\tilde{P}_{e} \tilde{\mathbf{b}}\right\rangle$ can be regarded as electron momentum (current) flux transported by magnetic fluctuations. ${ }^{31}$ In this sense, the diamagnetic dynamo term can include the kinetic dynamo effect which involves parallel pressure only. ${ }^{31}$ But the present measurement cannot distinguish whether or not the kinetic dynamo is present, which would require measurement of the parallel pressure fluctuations. The diamagnetic dynamo may be seen when the collision effects are included in the self-consistent kinetic theory.

\section{Relation with magnetic helicity}

The magnetic helicity ${ }^{32}$ is a quantity measuring the "knottedness" of magnetic field and is defined as $K=\int \mathbf{A} \cdot \mathbf{B} d V$ where $A$ is the vector potential. The helicity balance equation is given by

$$
\begin{aligned}
\frac{d K}{d t}= & -2 \int \mathbf{E} \cdot \mathbf{B} d V-2 \int \phi \mathbf{B} d \mathbf{S}-\int \mathbf{A} \times \dot{\mathbf{A}} d \mathbf{S}, \\
= & -2 \int \eta \mathbf{j} \cdot \mathbf{B} d V-2 \int \frac{\nabla P_{e} \cdot \mathbf{B}}{e n} d V-2 \int \phi \mathbf{B} d \mathbf{S} \\
& -\int \mathbf{A} \times \dot{\mathbf{A}} d \mathbf{S},
\end{aligned}
$$

where $\phi$ is the electrostatic potential and the generalized Ohm's law Eq. (2) is used. The first term in the RHS of the above equation is the helicity dissipation rate and the last term represents helicity injection rate.

The dynamo effect generates parallel current which is closely related to the magnetic helicity. The volume integral of the MHD dynamo can be rewritten as

$$
\int\left\langle\tilde{\mathbf{E}}_{\perp} \cdot \tilde{\mathbf{b}}_{\perp}\right\rangle d V \approx \int \boldsymbol{\nabla} \cdot\langle\tilde{\boldsymbol{\phi}} \tilde{\mathbf{b}}\rangle=\int\langle\tilde{\phi} \tilde{\mathbf{b}}\rangle d \mathbf{S},
$$

which corresponds to the third term in Eq. (10). Corresponding to the diamagnetic dynamo, the second term can be rewritten as

$$
\int \frac{\left\langle\nabla_{\perp} \tilde{P}_{e} \cdot \tilde{\mathbf{b}}_{\perp}\right\rangle}{e n} d V \approx \frac{1}{e n} \int\left\langle\tilde{P}_{e} \tilde{\mathbf{b}}\right\rangle d \mathbf{S}
$$

by using Eq. (8). Therefore, $\langle\tilde{\phi} \tilde{\mathbf{b}}\rangle$ is the helicity flux due to MHD dynamo effect while $\left\langle\tilde{P}_{e} \tilde{\mathbf{b}}\right\rangle$ is the helicity flux due to electron diamagnetic dynamo effect. The appearance as surface terms in the helicity balance equation means that both dynamo mechanisms transport the helicity across space and conserve the total helicity when they vanish at the surface.

In the typical astrophysical dynamo setting, the helicity monotonically grows in time. But in the laboratory plasmas, such as in the RFPs, the helicity is conjectured by Taylor ${ }^{33}$ to be conserved during the plasma relaxation in which the magnetic energy decays toward a minimum-energy state. The sawtooth crash phase in MST corresponds to this process which is associated with a large, discrete dynamo electric field as described in Sec. IV. The direct measurements ${ }^{34}$ of the helicity flux indicate that the prominent dynamo activity during the sawtooth crash transports magnetic helicity from center to edge, but the total helicity is roughly conserved, verifying Taylor's conjecture. 


\section{CONCLUSIONS}

Three major findings of the experiments in three laboratory plasmas are (1) the $\alpha$ effect accounts for the dynamo current generation, even in the time dependence of the parallel current through a sawtooth cycle; (2) at low collisionality the dynamo is explained primarily by the widely studied pressureless MHD model, i.e., the fluctuating velocity is dominated by the $\mathbf{E} \times \mathbf{B}$ drift; (3) at high collisionality, a new diamagnetic dynamo is observed, in which the fluctuating velocity is dominated by the electron diamagnetic drift. Both dynamo mechanisms transport magnetic helicity across space through a fluctuation-induced helicity flux but conserve the total helicity. The detailed transition mechanism toward the diamagnetic dynamo as well as its applicability to the astrophysical dynamos awaits future exploration.

\section{ACKNOWLEDGMENTS}

The authors are grateful to Professor P. Terry for valuable discussions.

This work was supported by the U.S. Department of Energy, Japanese Science and Technology Agency, and Japanese Ministry of Education.

\section{APPENDIX: TIME-DEPENDENT CORRELATION ANALYSIS}

The dynamo electric field arises from the correlation between two fluctuating quantities. Conventional spectral analysis provides an effective way to calculate cross correlation when fluctuations are stationary in time (or homogeneous in space). The cross correlation between two fluctuating quantities $\tilde{A}$ and $\tilde{B}$ is given by

$$
\begin{aligned}
\langle\tilde{A} \tilde{B}\rangle & =\int P_{A B}(f) \mathrm{d} f \\
& =\int|\tilde{A}(f) \| \tilde{B}(f)| \gamma_{A B}(f) \cos \theta_{A B}(f) \mathrm{d} f,
\end{aligned}
$$

where $P_{A B}$ is the cross-power spectrum, $|\tilde{A}|$ and $|\tilde{B}|$ are the fluctuation amplitudes, and $\gamma_{A B}$ and $\theta_{A B}$ are coherence and relative phase between $\tilde{A}$ and $\tilde{B}$, respectively. Since the plasma rotates in the laboratory frame, this method is equivalent to flux surface averaging even though the measurement position is fixed. Note here that the calculated quantities $P_{A B},|\tilde{A}|,|\tilde{B}|, \gamma_{A B}$ and $\theta_{A B}$ are functions of frequency and independent of time.

However, the above spectral method does not apply to the time-dependent phenomena, such as sawtooth oscillations in MST discharges. To obtain ensemble-averaged quantities (such as cross correlations) with time-resolved information during a sawtooth crash, an ensemble is constructed from time samples time-referenced to a crash. Suppose two measured quantities $A(t)$ and $B(t)$ are written as

$$
A(t)=\tilde{A}(t)+\bar{A}, \quad B(t)=\tilde{B}(t)+\bar{B},
$$

where the bar indicates the time average. The fluctuation parts can be written as

$$
\tilde{A}(t)=\tilde{A}^{R}(t)+\langle\tilde{A}\rangle(t), \quad \tilde{B}(t)=\tilde{B}^{R}(t)+\langle\tilde{B}\rangle(t),
$$

where the quantities with superscript $R$ denote random parts and $\langle\ldots\rangle$ the ensemble average, satisfying $\left\langle\tilde{A}^{R}\right\rangle \equiv\left\langle\tilde{B}^{R}\right\rangle \equiv 0$. For the rest of this section, the notation $(t)$ will be omitted since all quantities mentioned will be time-dependent. The ensemble average can consist of two components: symmetric component [i.e., $m=0, n=0$ component where $m(n)$ is poloidal (toroidal) mode number] and asymmetric component (i.e., $m \neq 0$ or $n \neq 0$ component). Therefore, if the ensemble average is equivalent to flux surface average (which is symmetric), then the symmetric components are zero.

The cross correlation between $\tilde{A}$ and $\tilde{B}$ is given by

$$
\tilde{A} \tilde{B}=\tilde{A}^{R} \tilde{B}^{R}+\tilde{A}^{R}\langle\tilde{B}\rangle+\langle\tilde{A}\rangle \tilde{B}^{R}+\langle\tilde{A}\rangle\langle\tilde{B}\rangle .
$$

Therefore, the time-dependent flux surface average of $\tilde{A}^{R} \tilde{B}^{R}$ becomes

$$
\left\langle\tilde{A}^{R} \tilde{B}^{R}\right\rangle=\langle\tilde{A} \tilde{B}\rangle-\langle\tilde{A}\rangle\langle\tilde{B}\rangle,
$$

where the last term is the correction due to symmetric (equilibrium) changes in $\tilde{A}$ and $\tilde{B}$ and incomplete flux surface average from the localization of the measurements. The time-dependent fluctuation amplitude can be obtained by taking $A \equiv B$ in Eq. (A5):

$$
\left|\tilde{A}^{R}\right|^{2} \equiv\left\langle\left(\tilde{A}^{R}\right)^{2}\right\rangle-\langle\tilde{A}\rangle^{2} .
$$

As an analogy to the spectral analysis, we can define the time-dependent coherence $\gamma(t)$ and phase difference $\theta(t)$ :

$$
\left\langle\tilde{A}^{R} \tilde{B}^{R}\right\rangle /\left|\tilde{A}^{R}\right|\left|\tilde{B}^{R}\right| \equiv \gamma(t) \cos \theta(t) \equiv a(t) .
$$

We can interpret them as "power-weighted" or "effective" coherence and phase difference between two fluctuations.

In order to separate $\gamma$ and $\theta$ in Eq. (A7), the phaseshifted fluctuations are employed:

$$
\tilde{B}^{R, \delta \theta}(t)=\mathrm{FFT}^{-1}\left(\operatorname{FFT}\left(\tilde{B}^{R}(t)\right) \cdot \exp i \delta \theta\right),
$$

where $\mathrm{FFT}^{-1}$ is the inverse transformation of the Fast Fourier Transformation (FFT) and $\delta \theta$ is the shifted phase for every frequency component. Note that if dispersion relation is linear, then the phase shift in time is equivalent to phase shift in space. By using the phase-shifted $\tilde{B}$ with $\delta \theta=\pi / 2$ in

$$
\left\langle\tilde{A}^{R} \tilde{B}^{R, \pi / 2}\right\rangle /\left|\tilde{A}^{R}\right|\left|\tilde{B}^{R, \pi / 2}\right| \equiv \gamma \cos (\theta+\pi / 2) \equiv b(t),
$$

and Eq. (A7) we have

$$
\gamma=\sqrt{a^{2}+b^{2}}, \quad \theta=\tan ^{-1}(-a / b) .
$$

${ }^{1}$ H. K. Moffatt, Magnetic Field Generation in Electrically Conducting Fluids (Cambridge University Press, Cambridge, 1978).

${ }^{2}$ For example, A. Brandenburg, in Lectures on Solar and Planetary Dynamos, edited M. R. E. Proctor and A. D. Gilbert (Cambridge University Press, Cambridge, 1994), p. 117.

${ }^{3}$ For example, an early simulation result is E. J. Caramana, R. A. Nebel, and D. D. Schnack, Phys. Fluids 26, 1305 (1983); and a recent one is A. Nagata, H. Sakamoto, K. I. Sato, A. Ashida, and T. Amano, Phys. Plasmas 2, 1182 (1995).

${ }^{4}$ E. J. Caramana and D. A. Baker, Nucl. Fusion 24, 423 (1984).

${ }^{5}$ A. Janos, Phys. Fluids 29, 3342 (1986).

${ }^{6}$ A. Gailitis, O. Lielausis, B. G. Karasev, I. R. Kirillov, and A. P. Ogorodnikov, in Liquid Metal Magnetohydrodynamics, edited by J. Lielpeteris and R. Moreau (Kluwer, Dordrecht, 1989), p. 413. 
${ }^{7}$ H. Ji, H. Toyama, A. Fujisawa, S. Shinohara, and K. Miyamoto, Phys. Rev. Lett. 69, 616 (1992).

${ }^{8}$ A. al-Karkhy, P. K. Browning, G. Cunningham, S. J. Gee, and M. G. Rusbridge, Phys. Rev. Lett. 70, 1814 (1993).

${ }^{9}$ A. Ejiri, S. Ohdachi, T. Oikawa, S. Shinohara, K. Yamagishi, H. Toyama, and K. Miyamoto, Phys. Plasmas 1, 1177 (1994).

${ }^{10}$ D. J. Den Hartog, A. F. Almagri, J. T. Chapman, H. Ji, S. C. Prager, and J. S. Sarff, Phys. Plasmas 2, 2281 (1995).

${ }^{11}$ H. Ji, A. F. Almagri, S. C. Prager, and J. S. Sarff, Phys. Rev. Lett. 73, 668 (1994).

${ }^{12}$ H. Ji, Y. Yagi, K. Hattori, A. F. Almagri, S. C. Prager, Y. Hirano, J. S. Sarff, T. Shimada, Y. Maejima, and K. Hayase, Phys. Rev. Lett. 75, 1086 (1995).

${ }^{13}$ L. Spitzer, Jr., Physics of Fully Ionized Gases, 2nd revised ed. (Interscience, New York, 1962), p. 28.

${ }^{14}$ See, e.g., E. N. Parker, Cosmical Magnetic Fields (Clarendon, Oxford, 1979).

${ }^{15}$ R. N. Dexter, D. W. Kerst, T. W. Lovell, S. C. Prager, and J. C. Sprott, Fusion Technol. 19, 131 (1991).

${ }^{16}$ N. Asakura, Y. Nagayama, S. Shinohara, H. Toyama, and K. Miyamoto, Nucl. Fusion 29, 893 (1989).

${ }^{17}$ Y. Yagi, P. R. Brunsell, Y. Hirano, T. Shimada, Y. Maejima, I. Hirota, and K. Sugisaki, in Plasma Physics and Controlled Nuclear Fusion Research 1992 (International Atomic Energy Agency, Vienna, 1993), Vol. 2, p. 661.

${ }^{18}$ S. Hokin, A. Almagri, S. Assadi, J. Beckstead, G. Chartas, N. Crocker, M. Cudzinovic, D. Den Hartog, R. Dexter, D. Holly, R. Nebel, S. Prager, T. Rempel, J. Sarff, E. Scime, W. Shen, C. Spragins, C. Sprott, G. Starr, M. Stoneking, and C. Watts, Phys. Fluids B 3, 2241 (1991).

${ }^{19}$ H. Ji, H. Toyama, K. Yamagishi, S. Shinohara, A. Fujisawa, and K. Miyamoto, Rev. Sci. Instrum. 62, 2326 (1991).
${ }^{20}$ A. F. Almagri, S. Assadi, S. C. Prager, J. S. Sarff, and D. W. Kerst, Phys. Fluids B 4, 4080 (1992).

${ }^{21}$ J. C. Ingraham, R. F. Ellis, J. N. Downing, C. P. Munson, P. G. Weber, and G. A. Wurden, Phys. Fluids B 2, 143 (1990).

${ }^{22}$ M. R. Stoneking, S. A. Hokin, S. C. Prager, G. Fiksel, H. Ji, and D. J. Den Hartog, Phys. Rev. Lett. 73, 549 (1994).

${ }^{23}$ Y. Yagi, Y. Hirano, Y. Maejima, P. R. Brunsell, T. Shimada, and K. Hattori, "Measurement of energy distribution of fast electrons by an electron energy analyzer in a reversed field pinch plasma on TPE-1RM20," submitted to Jpn. J. Appl. Phys.

${ }^{24}$ Y. Yagi, P. R. Brunsell, Y. Hirano, I. Hirota, Y. Maejima, and T. Shimada, "Fluctuations and Confinement in the TPE-1RM20 Reversed Field Pinch," in Plasma Physics and Controlled Nuclear Fusion Research, 1994 (International Atomic Energy Agency, Vienna, in press), Paper No. C-1I-3.

${ }^{25}$ M. G. Rusbridge, Plasma Phys. 11, 35 (1969).

${ }^{26}$ H. Y. W. Tsui, C. P. Ritz, G. Miller, J. C. Ingraham, C. P. Munson, K. F. Schoenberg, and P. G. Weber, Nucl. Fusion 31, 2371 (1991).

${ }^{27}$ W. Shen and S. C. Prager, Phys. Fluids B 5, 1931 (1993).

${ }^{28}$ S. I. Braginskii, in Reviews of Plasma Physics (Consultants Bureau, New York, 1965), Vol. 1, p. 205.

${ }^{29}$ A. R. Jacobson and R. W. Moses, Phys. Rev. A 29, 3335 (1984).

${ }^{30}$ P. W. Terry and P. H. Diamond, Phys. Fluids B 2, 1128 (1990).

${ }^{31}$ S. C. Prager, Plasma Phys. Controlled Fusion 32, 903 (1990).

${ }^{32}$ H. K. Moffatt, J. Fluid Mech. 35, 117 (1969).

${ }^{33}$ J. B. Taylor, Phys. Rev. Lett. 33, 1139 (1974); Rev. Mod. Phys. 58, 741 (1986).

${ }^{34}$ H. Ji, S. C. Prager, and J. S. Sarff, Phys. Rev. Lett. 74, 2945 (1995). 